\title{
PREGNANCY-ASSOCIATED BREAST CANCER
}

\section{https://doi.org/10.29289/259453942020V30S1085}

\section{GESTATIONAL GIGANTOMASTIA: A CASE SERIES}

Camila Vitola Pasetto', Natalia de Souza Costa', Isabela do Prado Nascimento1, Marcos Daniel Weffort ${ }^{1}$, Vinicius Milani Budel ${ }^{1}$

${ }^{1}$ Hospital de Clínicas da Universidade Federal do Paraná - Curitiba (PR), Brazil.

Introduction: Gestational gigantomastia (GG) is a rare condition known for the massive growth of one or both breasts during pregnancy. Objectives: to describe three cases of GG in patients assisted Hospital de Clínicas do Paraná. Case Reports: Case 1 - Twenty-four year old pregnant woman, of 9 weeks, presented with mastalgia and increasing breast volume associated with phlogistic signs and orange peel-like skin. Breast ultrasound showed diffuse inflammatory process. A biopsy was performed and showed ductal ectasia and stromal fibroadenomatoid changes. On the $20^{\text {th }}$ week, she was hospitalized with fever, voluminous breasts and drainage of purulent secretion and long ulcers in the inframammary fold. She was administered antibiotics and local care. The choice was to interrupt the pregnancy of 33 weeks. She presented with relative reduction of the breasts and breastfed, but had severe psychological problems caused by the aesthetic dissatisfaction. Case 2 - Eighteen-year old patient presented with infiltrating ductal breast carcinoma in the right breast, treated with sectorectomy, with sentinel lymph node and conventional radiotherapy. After one year of follow-up, ductal carcinoma in situ (DCIS) was diagnosed in the left breast, being submitted to sectorectomy and intraoperative radiotherapy. Two years after DCIS, she became pregnant and presented with GG only in the left breast, with remarkable asymmetry and skin edema. After the Cesarean section, breastfeeding was normal on the GG side, but not on the right side, due to a sequel from total radiotherapy. Case 3 - Twenty-two year old pregnant women, of 12 weeks, presented with mastalgia and exaggerated growth of the breasts, with and orange peel-like skin. Breast ultrasound showed bilateral inflammatory process. On the $15^{\text {th }}$ week, she presented with bleeding ulcers, right breast with latero-lateral diameter of $48 \mathrm{~cm}$, and craniocaudal diameter of $56 \mathrm{~cm}$, and left breast with $49 \mathrm{~cm}$ and $58 \mathrm{~cm}$, respectively. Due to the fast and progressive growth of the breasts, with difficulties to breath, major venous and lymph stasis and extensive skin ulceration/maceration, with risk of infection, the patient and relatives agreed on conducting a therapeutic abortion, followed by a reduction mammoplasty. Discussion: It is a rare disease, and its etiology remains unknown. Medicinal therapy seems to be first option, but it is often necessary to complement it with a surgical procedure. Conclusions: GG is a pathology of great morbidity, with tendency to recede in the puerperium. In case it is not possible to wait, the recommendation is to intervene in the pregnancy. 